\title{
On the Nonexistence of Simplex Integration Rules for Infinite Integrals
}

\section{By P. J. Davis and P. Rabinowitz}

Abstract. It is shown that there do not exist integration rules of the form

$$
\int_{0}^{\infty} f(x) d x=\sum_{i=1}^{n} w_{i} f\left(x_{i}\right)+C_{n} f^{(m)}(\xi), \quad 0<\xi<\infty
$$

Almost all classical integration rules over a finite interval are simplex, that is, they have the form

$$
\int_{a}^{b} f(x) d x=\sum_{i=1}^{n} w_{i} f\left(x_{i}\right)+C f^{(k)}(\xi), \quad a<\xi<b, \xi=\xi(f),
$$

where $C$ is a constant depending on the rule and interval, but independent of $f$, and $k$ is some integer which is characteristic for the rule. Some special rules, for example Weddle's rule, which are not simplex are multiplex, that is, the error has the form $\sum_{j=1}^{m} C_{i} f^{\left(k_{i}\right)}\left(\xi_{i}\right)$. It is the aim of this note to show that there can exist no simplex or multiplex rule for the infinite integral $\int_{0}^{\infty} f(x) d x$. Although the Gauss-Laguerre rule

$$
\int_{0}^{\infty} e^{-x} f(x) d x=\sum_{i=1}^{n} w_{i} f\left(x_{i}\right)+C_{n} f^{(2 n)}(\xi)
$$

appears to have the form of a simplex rule, this is not so, since we are concerned with unweighted integrals and if we write $f(x)=e^{-x} e^{x} f(x)$, we have that

$$
\int_{0}^{\infty} f(x) d x=\sum_{i=1}^{n} w_{i} e^{x_{i}} f\left(x_{i}\right)+C_{n}\left(e^{x} f(x)\right)_{x=\xi}^{(2 n)}
$$

which is neither simplex nor multiplex in form.

We now show that it is impossible to have an integration rule of the form

$$
\text { If } \equiv \int_{0}^{\infty} f(x) d x=\sum_{i=1}^{n} w_{i} f\left(x_{i}\right)+C f^{(k)}(\xi), \quad 0<\xi<\infty,
$$

valid for all $f \in L[0, \infty) \cap C^{k}(0, \infty)$, or for that matter, one where there are a finite number of terms of the form $C_{i} f^{\left(k_{j}\right)}\left(\xi_{i}\right)$. The proof is based on the simple fact that, for any $r>0$,

$$
\int_{0}^{\infty} f(x) d x=r \int_{0}^{\infty} f(r x) d x
$$

Received February 7, 1972.

AMS 1970 subject classifications. Primary 41A55; Secondary 65D30.

Key words and phrases. Numerical integration, infinite interval, simplex rule, integration error.

Copyright (c) 1972, American Mathematical Society 
If (1) were true, then (2) would imply that

(3)

$$
\begin{aligned}
I f & =r \int_{0}^{\infty} f(r x) d x=r \sum_{i=1}^{n} w_{i} f\left(r x_{i}\right)+r C f^{(k)}(r \xi) \\
& =r \sum_{i=1}^{n} w_{i} f\left(r x_{i}\right)+r^{k+1} C f^{(k)}(\xi), \quad 0<\xi<\infty,
\end{aligned}
$$

which must hold for all $f \in L[0, \infty) \cap C^{k}(0, \infty)$ and any real $r$. If we now choose such a function which is bounded together with its $k$ th derivative on $[0, \infty)$, say $f(x)=1 /\left(1+x^{2}\right)$, and let $r$ approach zero, we see that the right-hand side of (3) approaches zero while the left-hand side has a constant value. This contradiction proves our result.

\section{Brown University}

Providence, Rhode Island 02912

Weizmann Institute of Science

Rehovot, Israel 